\title{
Primary cilia presence and implications in bladder cancer progression and invasiveness
}

\author{
Pablo Iruzubieta ${ }^{1,2}$ (1) Tomás Castiella ${ }^{2,3}$ (1) Eva Monleón ${ }^{1} \cdot$ Carmen Berga $^{1,2} \cdot$ Guillermo Muñoz $^{3} \cdot$ \\ Concepción Junquera ${ }^{1,2} \mathbb{0}$
}

Accepted: 13 January 2021

(c) The Author(s), under exclusive licence to Springer-Verlag GmbH, DE part of Springer Nature 2021

\begin{abstract}
Urothelial bladder cancer is the tenth most common cancer worldwide. It is divided into muscle and non-muscle invading bladder cancer. Primary cilia have been related to several cancer hallmarks such as proliferation, epithelial-to-mesenchymal transition (EMT) or tumoral progression mainly through signaling pathways as Hedgehog (Hh). In the present study, we used immunohistochemical and ultrastructural techniques in human tissues of healthy bladder, non-muscle-invasive bladder cancer (NMIBC) and muscle-invasive bladder cancer (MIBC) to study and clarify the activation of epithelial-to-mesenchymal transition and Hedgehog signaling pathway and the presence of primary cilia. Thus, we found a clear correlation between EMT and Hedgehog activation and bladder cancer stage and progression. Moreover, we identified the presence of primary cilia in these tissues. Interestingly, we found that in NMIBC, some ciliated cells cross the basement membrane and localized in lamina propria, near blood vessels. These results show a correlation between EMT beginning from urothelial basal cells and primary cilia assembly and suggest a potential implication of this structure in tumoral migration and invasiveness (likely in a Hh-dependent way). Hence, primary cilia may play a fundamental role in urothelial bladder cancer progression and suppose a potential therapeutic target.
\end{abstract}

Keywords Bladder cancer · Primary cilia $\cdot$ Epithelial-mesenchymal transition $\cdot$ Hedeghog signalling pathway $\cdot$ Ultrastructure $\cdot$ Transmission electron microscopy

\section{Introduction}

Bladder cancer is the tenth most common cancer worldwide (Global Cancer Observatoty 2018), being three times more frequent in men than women (Antoni et al. 2017). In the Western countries, the most common histological subtype is urothelial cancer, reaching $90 \%$ of cases (Bryan 2015).

Supplementary Information The online version contains supplementary material available at https://doi.org/10.1007/s0041 8-021-01965-2.

Pablo Iruzubieta

pablo.iruzubieta@hotmail.es

1 Department of Human Anatomy and Histology, Faculty of Medicine, University of Zaragoza, Zaragoza, Spain

2 Institute for Health Research Aragón (IIS), Domingo Miral s/n, 50009 Zaragoza, Spain

3 Department of Pathology, Hospital Clínico Universitario Lozano Blesa, Zaragoza, Spain
Urothelial tumours are mainly classified in non-invasive and infiltrating neoplasms regarding the absence or presence (respectively) of muscle invasion (Humphrey et al. 2016). Non-Muscle Invasive Bladder Cancer (NMIBC) tumours comprise $75-85 \%$ of all urothelial cancers while the remaining 15-25\% are Muscle Invasive Bladder Cancers (MIBC), which cause most bladder cancer-related deaths (Bryan 2015). Although NMIBC survival rate is excellent, it shows frequent recurrence and progression to MIBC (50\% and 9\%, respectively) (Liedberg et al. 2015).

Carcinomas progression and invasiveness are closely related to epithelial-to-mesenchymal transition (EMT) (Prieto-García et al. 2017). EMT consists of the dynamic and reversible switch from an epithelial non-motile to a mesenchymal motile phenotype (Zeisberg and Neilson 2009; Lamouille et al. 2014; Prieto-García et al. 2017). During EMT, epithelial tumor cells undergo transient changes resulting in a mesenchymal phenotype characterized by a loss of cellular junctions, changes in cell-matrix adhesion, loss of apicobasal polarity, cytoskeletal changes and an increased 
contractility and motility (Lamouille et al. 2014). These features allow tumor cells to release from neighbor cells and migrate to distant areas. Thus, EMT is a key process in tumor progression, invasiveness and metastasis (Zeisberg and Neilson 2009; Prieto-García et al. 2017). EMT is induced by several transcription factors (remarkably Snail1, Snail2 or Slug, Twist, ZEB1 and ZEB2) (Lamouille et al. 2014). These transcription factors repress the expression of epithelial-related proteins such as cytokeratin and E-cadherin (which mediates cell to cell junctions) (Lamouille et al. 2014). Conversely, they induce the transcription of genes such as Vimentin and N-cadherin, which confer a mesenchymal phenotype (Lamouille et al. 2014). Regarding bladder cancer, Twist is the best-studied EMT-related transcription factor and its expression has been related to smoking, poorer prognosis and increase metastasis (Fondrevelle et al. 2009; Yun and Kim 2013).

EMT can be triggered by different signalling pathways such as TGF $\beta$, Notch, Wnt or Hedgehog (Hh) (Lamouille et al. 2014). Most of these pathways have been related to primary cilia (Wheway et al. 2018). Primary cilium is an organelle composed of a basal body (originated from a modified centriole) which gives rise to an axoneme protruding outside the cell. It has been related to different cellular functions including signaling pathway activation, cytoskeletal functions, cell cycle control or protein homeostasis (Malicki and Johnson 2017). Moreover, this structure regulates several cancer hallmarks (Fabbri et al. 2019). However, the presence and importance of primary cilia in bladder cancer is still not known and it has just started to be explored (Du et al. 2018).

$\mathrm{Hh}$ is the best-studied cilia-dependent pathway and it has been closely related to several types of cancer (Rohatgi et al. 2007; Amakye et al. 2013; Castiella et al. 2013; Chahal et al. 2018; Iruzubieta et al. 2019). Hh proper activation depends on primary cilia (Rohatgi et al. 2007). Thus, when Hh ligands bind Patched receptor, located in the ciliary membrane, it is removed from the cilia allowing Smoothened (Smo) entrance to the membrane and Glioma associated oncogene 1 (Gli1) activation and translocation into cell nuclei (Rohatgi et al. 2007; Ramsbottom et al. 2016). Gli1 activates target genes transcription, including genes related to EMT, angiogenesis and cancer stem cell maintaining (Katoh and Katoh 2009; Amakye et al. 2013; Chahal et al. 2018).

The exact relationship between Hh pathway and bladder cancer remains elusive. Thus, Hh has been proposed both as an inductor of EMT and invasiveness (Islam et al. 2016; Nedjadi et al. 2019) and as a protector element that restrains bladder cancer progression (Shin et al. 2014b).

In the present study, we study through immunohistochemical techniques EMT activation, Hh signalling pathway and primary cilia presence in bladder cancer. Besides, we performed a detailed ultrastructural study of tumoral cell features and EMT process. Our results emphasize the importance of primary cilia in cancer metastasis and recurrence, proposing the relevance of primary cilia as a potential target in bladder cancer.

\section{Methods}

\section{Materials}

Six MIBC (all G3 except for one G2-G3, five pT2NxM0 and one pT3bN0M0), seven NMIBC (five G2 and two G1, all of them pTaNxM0) and two healthy bladder controls were used for the study. They were retrieved from the files of the Pathology Department at the University Clinic Hospital of Zaragoza. All protocols developed were approved by the Human Research Ethics Committee "Comité Ético de Investigación Clínica de Aragón".

\section{Electron microscopy}

Samples were washed in phosphate buffer and fixed with $2.5 \%$ glutaraldehyde and $2 \%$ paraformaldehyde overnight at $\mathrm{RT}$, washed in $0.1 \mathrm{M}$ phosphate buffer for $5 \mathrm{~min}$, post-fixed with $2 \%$ osmium, rinsed, dehydrated in graded ethanol (30\%, $50 \%, 70 \%$ with $2 \%$ uranyl acetate, $90 \%, 100 \%$ ), cleared in propylene oxide and embedded in Araldite (Durcupan, Fluka AG; Buchs SG, Switzerland).

Semi-thin sections $(1.5 \mu \mathrm{m})$ were cut with a diamond knife, lightly stained with $1 \%$ toluidine blue and examined by light microscopy (Olympus BX51 microscope, Olympus Imaging Corporation; Tokyo, Japan). Later, ultrathin $(0.05 \mu \mathrm{m})$ sections were cut, collected on Formvar coated single-slot grids counterstained with $1 \%$ uranyl acetate and Reynold's lead citrate for $1 \mathrm{~min}$.

They were examined under a JEOL 1010 TEM. The images were captured with Gatan Bioscan Camera with pixel size $6.45 \mu \mathrm{m} \times 6.45 \mu \mathrm{m}$ and spatial resolution of 1024,1024 , $1,1(\mathrm{x}, \mathrm{y}, \mathrm{z}, \mathrm{t})$ and automatic gain.

\section{Immunohistochemistry}

Immunohistochemical staining was performed on 4- $\mu \mathrm{m}$-thick formalin-fixed paraffin-embedded sections using EnVision ${ }^{\circledR}(\mathrm{DAKO})$ method. The primary antibodies used in this study were mouse anti-Cytokeratin Clone MNF116 (1:100, M0821 DAKO; Glostrup, Denmark), mouse anti-ECadherin Clone NCH-38 (1:100, M3612 DAKO; Glostrup, Denmark), mouse anti-Twist 2C1a (1:100, sc-81417, Santa Cruz, Dallas, TX, USA), rabbit anti-Gli1 (1:200, Abcam, ab49314; Cambridge, UK), rabbit anti-Smo (1:1000, LS Bioscience, LS-B4911, Seattle, WA, USA) and goat antiPatched (1:100, Santa Cruz, sc-6149; Dallas, TX, USA). 
The tissue sections were deparaffinised in xylene for $20 \mathrm{~min}$ and rehydrated in graded ethanol (see above). Prior to all assays, a heat-induced antigen retrieval step was performed for E-Cadherin, Gli1 and Smo immunolabeling using buffer citrate (pH6, DAKO S2031) by treatment for $5 \mathrm{~min}$ in microwave at $800 \mathrm{~W}$ and 3 additional minutes at $360 \mathrm{~W}$. For cytokeratin and patched staining, an antigen retrieval step using TBS (pH9) was performed at $96{ }^{\circ} \mathrm{C}$ for $20 \mathrm{~min}$. Twist unmasking was performed by heating in Tris-EDTA buffer (pH9) at $98^{\circ} \mathrm{C}$ for $40 \mathrm{~min}$.

After washing twice with PBS for $3 \mathrm{~min}$, endogenous peroxidase was blocked using peroxidase blocking reagent (DAKO, S2001) for $10 \mathrm{~min}$ and sections were washed in distilled water and PBS 3 min, twice.

Afterwards, sections were incubated with primary antibodies at $4{ }^{\circ} \mathrm{C}$ overnight in a humidified chamber. Sections were washed in PBS three times for 5 min and incubated with Labelled Polymer EnVision-HRP (DAKO K5007) or Rabbit Anti-Goat Immunoglobulins/HRP (1:200, DAKO, P016002-2) for $1 \mathrm{~h}$. To confirm the presence of immunocomplexes, 3,3' diaminobenzidine was used as the chromogen. The samples were contrasted with Mayer's haematoxylin $7 \mathrm{~min}$, washed in tap water for $15 \mathrm{~min}$, dehydrated in graded ethanol $\left(2 \mathrm{~min}\right.$ in $70^{\circ}, 2 \mathrm{~min}$ in $96^{\circ}$ and $5 \mathrm{~min}$ in $100^{\circ}$ ), cleared in xylene and coverslipped with Eukitt (03,989 Sigma-Aldrich; St. Louis, MO, USA). Negative controls were based on samples to which no primary antibody was added. All antibodies were commercial and supposedly validated.

Whole samples were analysed and digital images from representative areas were captured by Olympus BX53 and Olympus UIS UPLFLN Series objectives. FN: 26.5. $20 \times$ AN 0.5 and $40 \times A N 0.75$. Images were captured with Olympus DP72 Digital Camera. Pixel size $6.45 \mu \mathrm{m} \times 6.45 \mu \mathrm{m}$, total number of pixel 1.5 million. Exposition mode: SFL Auto and spatial resolution of $4140 \times 3096 \times 1 \times 1(\mathrm{x}, \mathrm{y}, \mathrm{z}, \mathrm{t})$.

\section{Immunofluorescence microscopy}

Four $\mu \mathrm{m}$ formalin-fixed paraffin-embedded sections were cut and mounted on glass slides. The sections were deparaffinised in xylene for $20 \mathrm{~min}$ and rehydrated in graded ethanol ( $4 \mathrm{~min}$ in $100^{\circ}, 4 \mathrm{~min}$ in $96^{\circ}, 4 \mathrm{~min}$ in $70^{\circ}$ and $5 \mathrm{~min}$ in distilled water). Tissues were permeabilised with $0.1 \%$ Triton $\mathrm{X}-100$ in PBS for $8 \mathrm{~min}$. Afterwards, an antigen retrieval step using Tris-buffered saline (TBS, pH9) was performed at $96{ }^{\circ} \mathrm{C}$ for $20 \mathrm{~min}$.

Sections were incubated overnight at $4{ }^{\circ} \mathrm{C}$ with the following primary antibodies: mouse anti-Acetylated tubulin (1:4000, Sigma-Aldrich, T7451; St. Louis, MO, USA) and rabbit anti-Pericentrin (1:100, Abcam, ab4448; Cambridge, UK) in a dark humidified chamber. Sections were washed in PBS three times for $5 \mathrm{~min}$ and incubated for $1 \mathrm{~h}$ at RT in a dark humidified chamber with secondary antibodies: donkey anti-mouse Alexa Fluor 594 (1:1000, ThermoFisher, R37115; Waltham, MA, USA), donkey anti-rabbit Alexa Fluor 488 (1:1000, ThermoFisher, A-21206; Waltham, MA, USA). After washing in PBS, DAPI $(1 \mu \mathrm{g} / \mathrm{mL}$, Sigma-Aldrich) was added for $1 \mathrm{~min}$ for nuclei counterstaining. Sections were washed in PBS and the slides were covered with fluorescence mounting medium (DAKO, S3023). Negative controls were based on samples to which no primary antibody was added. On the other hand, we used a ciliated tissue (Fallopian tube) as a positive control for cilia immunofluorescence. All antibodies were commercial and supposedly validated.

Images were analysed using the following filters: U-MNUA2 for DAPI, U-MWIABA2 for Alexa Fluor 488 and U-MWIG2 for Alexa Fluor 555 in a Olympus BX53 microscope and Olympus UIS UPLFLN Series objectives. FN: 26.5. $20 \times$ AN 0.5 and $40 \times$ AN 0.75. Images were captured with Olympus DP72 Digital Camera. Pixel size $6.45 \mu \mathrm{m} \times 6.45 \mu \mathrm{m}$, total number of pixel $1.5 \mathrm{mil}$ lion. Exposition mode: auto and spatial resolution of $4080 \times 3072 \times 1 \times 1(x, y, z, t)$.

Images were analysed with DP Controller Software. Every fluorescent channel was individually photographed using automatic autoexposure and channels were merged using FIJI Image_J software, where contrast and brightness were adjusted (Schindelin et al. 2012).

Statistical analyses were performed counting ciliated cells per High Power Field $(\mathrm{HPF}=\times 400)$. The areas analysed were representative of the samples. A non-parametric test (Kruskal-Wallis test) was used.

\section{Results}

\section{Histological study}

Haematoxylin-eosin staining was performed in all samples (Fig. 1). Healthy bladder controls showed four to eight cellular layers with the characteristic umbrella cells on top (Fig. 1a). NMIBC presented abundant papillae composed of a thin vascularized stromal core surrounded by an increased number of cellular layers (Fig. 1b). On the other hand, MIBC samples show abundant heterogenous cells with atypia features such as enormous nuclei with prominent nucleoli, pale vacuolized cytoplasm, numerous mitosis and muscle invasion by tumoral cell cords (Fig. 1c). In some cases, necrotic areas were found, indicative of uncontrolled proliferation (Fig. 1d). 
Fig. 1 Haematoxylin-eosin staining. a Healthy bladder composed of four to eight cellular layers and a prominent stroma. b NIMBCs show papillae of vascular stroma surrounded by numerous layers of tumoral cells that do not cross the basement membrane. c MIBCs are composed of heterogeneous atypical tumoral cells $(\mathrm{t})$ that invade muscle $(\mathrm{m})$. d Detail of MIBC showing mitosis (mi) and areas of necrosis (n). v: blood vessel. Scale bar $=200 \mu \mathrm{m}$
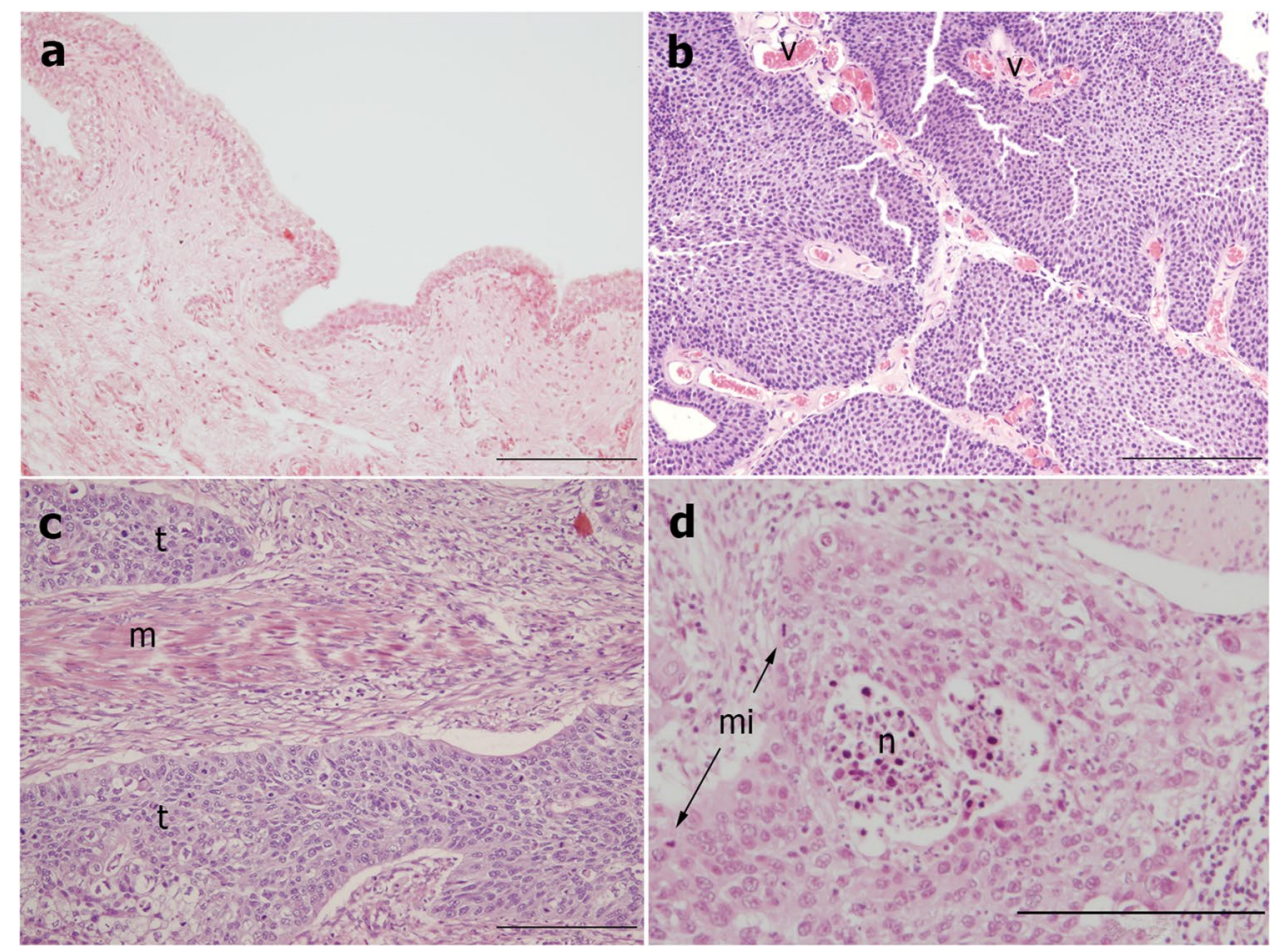

\section{Epithelial-to-mesenchymal transition (EMT) in bladder cancer progression}

As EMT is a predominant mechanism in tumor progression and invasiveness, we analyzed the expression of epithelial markers E-cadherin and Cytokeratin and the EMT-transcription factor Twist in bladder cancer stages regarding normal tissue (Fig. 2).

Our results showed that control samples showed prominent staining for cytokeratin (Fig. 2a) that was progressively reduced in NMIBC (Fig. 2b) and insignificant in MIBC (Fig. 2c). Similar results were found regarding E-cadherin expression (Fig. 2d-f). Although it was not consistently presented in the whole sample, there were specific areas in NMIBC tissues where E-cadherin and Cytokeratin expression was clearly reduced in basal cell layers and progressively increased in distal layers (Fig. 2b and e).

Accordingly, Twist nuclear staining was inexistent in the healthy bladder (Fig. 2g) and progressively increased and more intense in NMIBC (Fig. 2h) and MIBC (Fig. 2i). Twist expression was limited to tumoral cells while surrounding tissue showed no staining. The nuclear presence of Twist in these samples points to the activation of EMT program in bladder cancer cells, especially in MIBC. Once again, in NMIBC we found subtly predominant staining in basal layers (Fig. 2h).

Hence, our experiments confirmed that epithelial markers are reduced in bladder cancer while nuclear translocation of the EMT transcription factor Twist is increased. These findings confirmed that EMT activation correlates with tumoral grade. Moreover, we found that NMIBC samples show EMT indicators predominantly in basal layers.

\section{Hh signalling pathway in bladder cancer progression}

Afterwards, we studied the presence of different Hh signalling pathway components in different tumor stages to clarify the role of this pathway in bladder cancer.

Thus, we performed immunohistochemical experiments for Patched receptor, Smo and the main pathway effector Gli1 (Fig. 3). We found that Hh pathway components were practically no present in healthy controls while they show intermediate staining in NMIBC and they were prominently expressed in MIBC.

Smo expression was absent in healthy controls (Fig. 3a) and subtle expression was found in NMIBC (Fig. 3b). However, in MIBC, Smo staining was present in cell membrane and intense cytoplasm accumulations (Fig. 3c). Accordingly, Patched was not expressed in control bladders (Fig. 3d). Conversely, it was found in a localized paranuclear area both in NMIBC (Fig. 3e) and MIBC (Fig. 3f), being especially intense and abundant in the latter.

Gli1 nuclear staining is an indisputable sign of Hh signaling pathway activation. No Gli1 staining was found in healthy controls (Fig. 3g), but light presence appeared in several NMIBC cells (Fig. 3h). Some isolated positive cells 


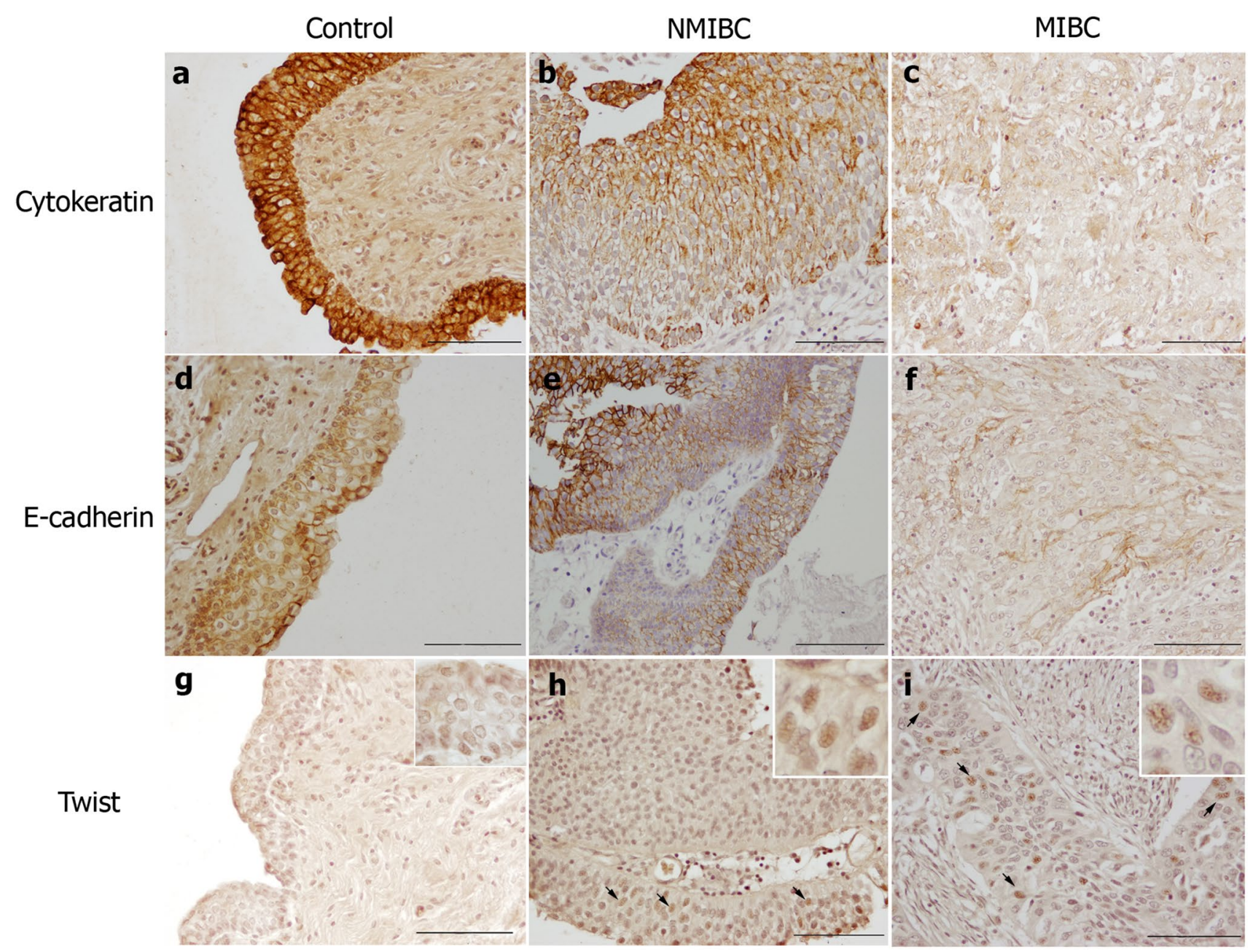

Fig. 2 Epithelial-to-mesenchymal transition (EMT) in bladder cancer. Reduction of epithelial markers and nuclear location of Twist are signs of EMT. a Cytokeratin cytoplasmic expression was intensely positive in healthy controls. b In non-invasive tumours (NMIBC), some areas with characteristic staining were found. These areas showed a reduced cytokeratin expression in basal layers. c In muscle-invasive bladder cancer (MIBC), however, only a weak expres-

were found beyond the basement membrane. Gli1 nuclear expression was intensely present in MIBC cells (Fig. 3i).

These results show that $\mathrm{Hh}$ activation is directly related to tumoral grade. This supports the hypothesis of $\mathrm{Hh}$ signalling pathway role in bladder cancer progression and invasiveness showing, consequently, an intense expression in high-grade tumours.

\section{Primary cilia in bladder cancer}

Canonical Hh signalling pathway is mediated by primary cilia both in physiology and cancer. Once we found Hh activation in bladder cancer, we studied the presence of primary cilia in these tumours through immunofluorescence techniques. Hence, using specific markers for both primary cilia sion was found. d-f Similar findings appeared regarding E-cadherin expression, which was mainly located in cell membranes. $\mathbf{g}$ Twist nuclear staining was fundamentally negative in control bladders, $\mathbf{h}$ whereas it was weakly positive in NMIBC especially in basal layers, although also distally and $\mathbf{i}$ clearly positive in numerous tumoral cells of MIBC but no in the surrounding tissue. Scale bar $=100 \mu \mathrm{m}$

axoneme (Acetylated Tubulin) and centrioles (Pericentrin), we analyzed the presence, number and proportion of primary cilia in healthy bladders, NMIBC and MIBC (Fig. 4).

Primary cilia were found in controls (Fig. 4a-c), NMIBC (Fig. 4d-f) and MIBC (Fig. 4g-i). Interestingly, both healthy controls and NMIBC, showed ciliated cells located fundamentally (though not uniquely) in basal layers (Fig. 4a and d). Furthermore, several ciliated cells were found across the basement membrane in NMIBC (Fig. 4d). Quantitative analyses were performed counting ciliated cells per high power field and a non-parametric test (Kruskal-Wallis) was used (Fig. 4j). The number of ciliated cells showed a clear tendency to progressively increase from controls to MIBC, although no statistical significance was found $(p=0.084)$. As these differences could be secondary to the increased 


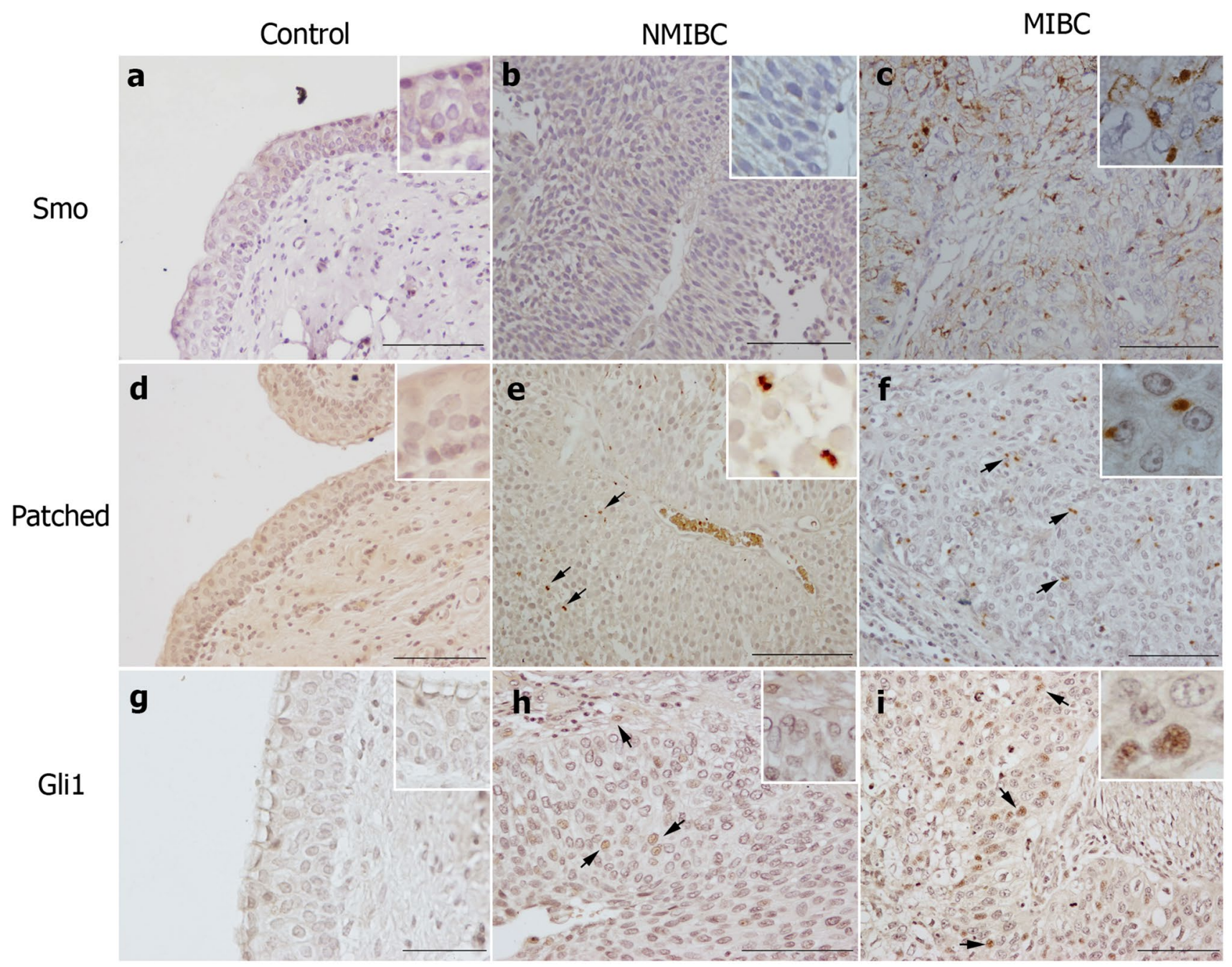

Fig. $3 \mathrm{Hh}$ signaling pathway activation in bladder cancer. Immunohistochemical staining for main $\mathrm{Hh}$ pathway components (Smo, Patched and Gli1) was performed. a Control bladders show no expression of Smo. b Non-muscle-invasive bladder cancer (NMIBC) showed weak positivity to Smo while c Muscle-invasive bladder cancer (MIBC) showed intense expression both in cell membrane and cytoplasm aggregation. d Patched was not found in control tissues.

number of cells found in cancer, we also studied the proportion of ciliated cells regarding the total number of cells per high power field (Fig. 4k). No apparent nor statistical significant differences were found neither $(p=0.4264)$.

In conclusion, primary cilia were found in healthy controls, NMIBC and MIBC with a non-statistically significant tendency to be increased in high-grade tumours.

\section{Ultrastructural study}

To better characterize the cells and the presence of primary cilia in bladder cancer, ultrastructural studies using transmission electron microscopy (TEM) were performed in tumoral tissues. e Subtle expression was present in NMIBC and $\mathbf{f}$ intense positivity was found in MIBC, showing paranuclear aggregates. g Regarding Gli1, no nuclear staining was found in controls. h Some subtly positive cells were found in NMIBC, Isolated Gli1 + cells were found beyond the basement membrane. i Intense nuclear expression was identified in MIBC tumoral cells but not in surrounding cells. Scale bar $=100 \mu \mathrm{m}$

\section{NMIBC}

In low-grade tumours, hyperplasic epithelial cells were found. They appeared organized in perpendicular layers to the papillae axe. The number of cellular layers was increased comparing healthy controls (Fig. 5a). Two cellular subtypes were clearly identified regarding cytoplasm electron density: clear and dark cells (Fig. 5b).

On the one hand, clear cells were less numerous and they showed less electron dense cytoplasm. These cells were characterized by scarce rough endoplasmic reticula cisterns close to dictyosomes of Golgi apparatus, free polyribosomes and paranuclear location of centrioles. Converse to dark cells, clear cells showed abundant mitochondria with 
Fig. 4 Primary cilia in controls, NMIBC and MIBC. Acetylated Tubulin marks ciliary axoneme while Pericentrin labels centrioles and, consequently, basal bodies. a-c Healthy bladder showing primary cilia in a general view (a) and detailed cells $(\mathbf{b}, \mathbf{c})$. Primary cilia were especially found in basal layers. d-f Primary cilia in NMIBC. Some ciliated cells were found across the basement membrane (dashed line). g-f Primary cilia in MIBC. j Quantitative analyses of total number of ciliated cells per High-Power Field. Apparent but not statistically significant differences could be detected by Kruskal-Wallis test $(p=0.084)$. $\mathbf{k}$ Proportion of ciliated cells regarding the total number of cells. No differences were found $(p=0.4264)$. Scale bar $=50 \mu \mathrm{m}$

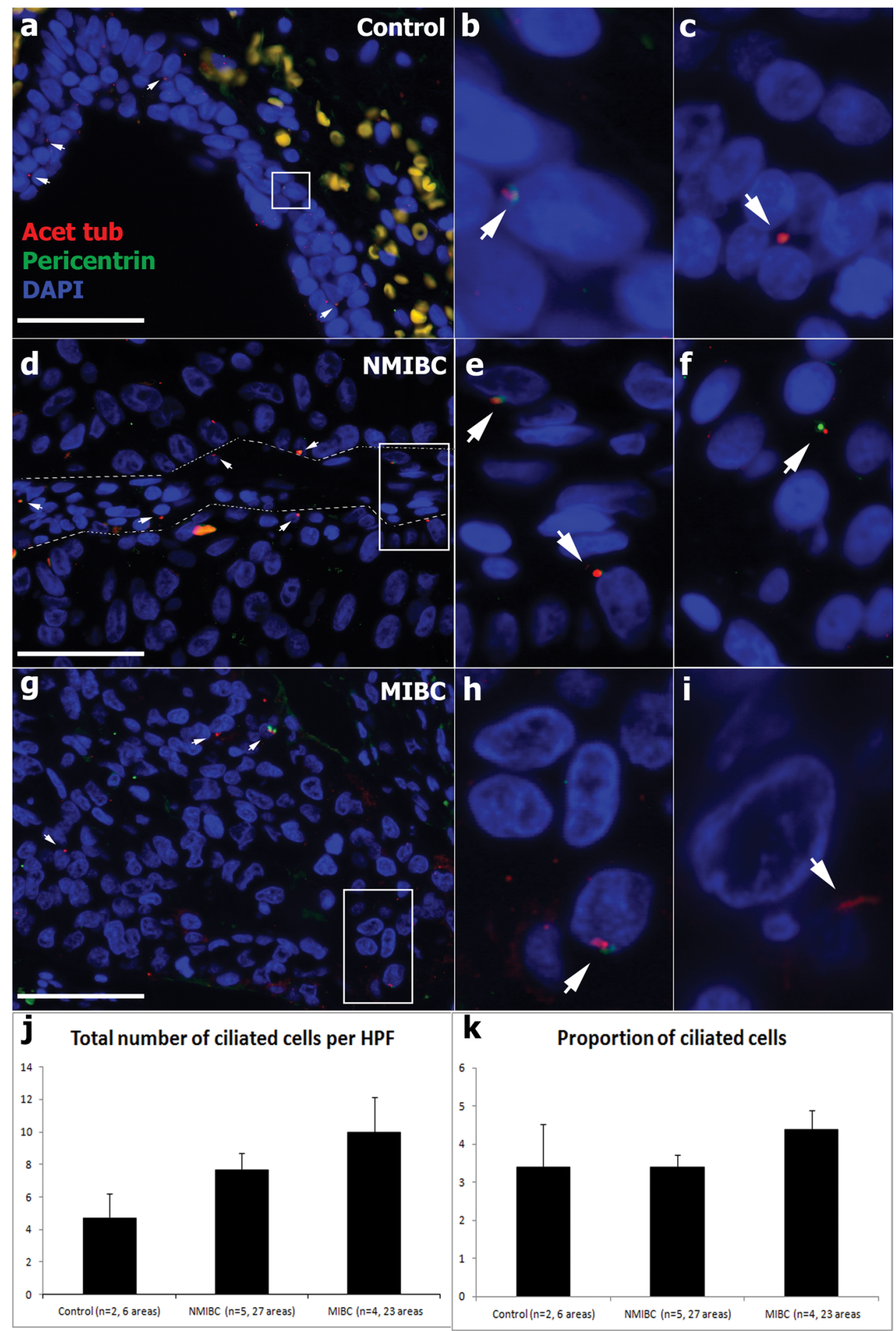

thicker crests (Fig. 5c). Moreover, they showed electron dense nuclei with abundant condensed heterochromatin, a sign of low transcriptional activity.

On the other hand, dark cells were the most frequent cell type in NMIBC. They showed voluminous oval nuclei with very decondensed euchromatin, suggesting active transcription, and a fine band of marginal heterochromatin surrounding the nuclear envelope. Nuclear envelopes characteristically presented deep invaginations (Fig. 5d) which sometimes generates interesting structures called nuclear envelope-limited chromatin sheets (ELCS) (Sup. Fig. 1a). Furthermore, they usually showed a prominent fibrillar nucleolus (Fig. 5d). Dark cells had a medium electron dense cytoplasm which contained similar organelles than clear cells (Fig. 5e). Especially relevant is the presence of multivesicular bodies fulfilled by vesicles of different 
Fig. 5 NMIBC ultrastructure. a Increased cellular layers in NMIBC. Scale bar $=20 \mu \mathrm{m}$ b Two cellular types were found in NMIBC based on cytoplasm electron density: clear cells (c) and dark cells (d). Scale bar $=5 \mu \mathrm{m}$ c Clear cells sowed condensed heterochromatin and a clear cytoplasm composed of scared rough reticulum, Golgi's dictyosomes (g) and small thick-crest mitochondria (m). Scale bar $=1 \mu \mathrm{m}$ d Dark cells, however, show decondensed euchromatin with fibrillar nucleoli (n) and deep nuclear envelope invaginations. Scale bar $=1 \mu \mathrm{m}$ e-f Dark cells cytoplasm is more electron dense and show abundant Golgi's dictyosomes (g), mitochondria (m) and characteristic multivesicular bodies (mvb). ct centrioles. Scale bar $=1 \mu \mathrm{m}$
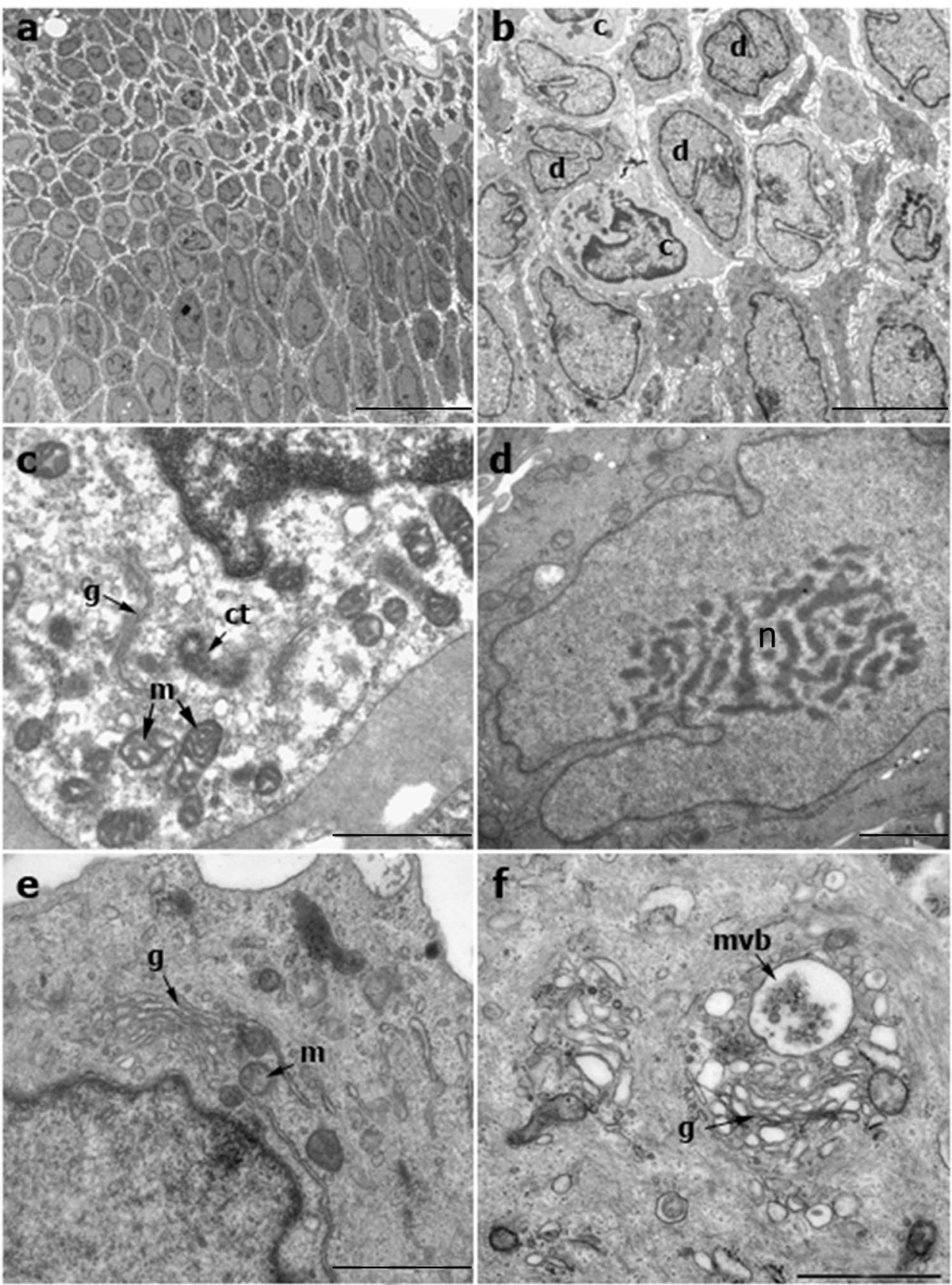

sizes (Fig. 5f). Abundant filaments originate from the characteristic darker cytoplasm.

A detailed ultrastructural study identified EMT features in NMIBC (Fig. 6). Hence, urothelial cells located in basal layers lost their apicobasal polarity (Fig. 6a) due to desmosomes disassembly (Fig. 6b) and formation of filopodialike structures (Fig. 6c), which allow cellular migration. As a consequence, intercellular space was wider. This mesenchymal phenotype acquisition was related to an increased number of mitochondria and centrioles activation.

Thus, in some dark cells, activation of centrioles was found, characterised by the loss of their perpendicular disposition, the presence of ciliary vesicles and migration to the cellular surface (Fig. 7a). There, they bind the cell membrane and formed primary cilia (Fig. 7b, c). This structure was fundamentally found in basal cells (Fig. 7c). Primary cilia subcomponents such as $9+0$ axoneme, basal body, transition fibres binding the cell membrane and subdistal appendages were identified. Primary cilia base was ensheathed by a membrane invagination called cilliary pocket.

Although diagnosed as NMIBC, some cells were found invading the basement membrane, showing a clear deformation of the cellular shape and a nuclear hourglass 
Fig. 6 Ultrastructural changes in EMT in NIMBC. a Loss of polarity occurs in basal cells. Moreover, some dark cells (circled) have crossed the basement membrane. Scale bar $=10 \mu \mathrm{m} \mathbf{b}$ Tumoral cells lose desmosomes (inset) and initiate the formation of filopodia (fil). Scale bar $=2 \mu \mathrm{m}$ (inset $250 \mathrm{~nm}$ ) c Intercellular space is increased and cells show abundant filopodia (fil). $b v$ blood vessel. Scale bar $=2 \mu \mathrm{m}$
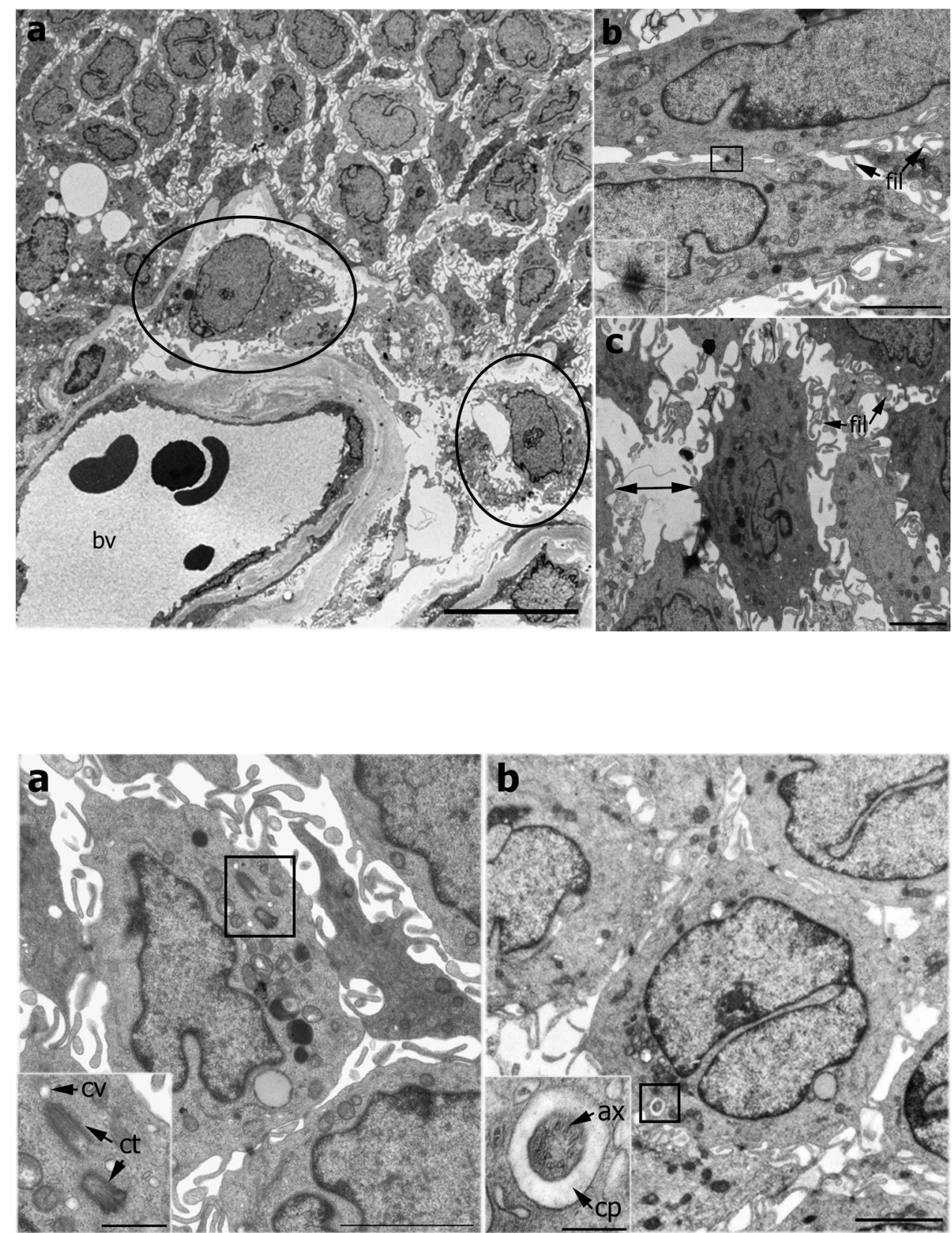

Fig. 7 EMT and primary cilia in dark cells of NMIBC and basement membrane invasion. a Dark cells located in the basal layer show activated centrioles which lost their typical perpendicular disposition (inset). Scale bar $=2 \mu \mathrm{m}$ (inset $500 \mathrm{~nm}$ ) b Activated centrioles originate primary cilia that emerges to the extracellular space (inset). Characteristic nuclear invaginations are identified in these cells. Scale bar $=2 \mu \mathrm{m}$ (inset $250 \mathrm{~nm})$ c A tumoral dark cell located on top of basement membrane showing an activated centriole binding cell membrane through transition fibres (tf) (inset). Scale bar $=5 \mu \mathrm{m}$ (inset $500 \mathrm{~nm}$ ) d Some tumoral cells crossed the basement membrane (bm) by adapting the cellular shape through an hourglassmorphology nucleus (arrows). Scale bar $=2 \mu \mathrm{m}$ ax axoneme, $c v$ ciliary vesicle, $c t$ centrioles, $c p$ ciliary pocket, $b b$ basal body, $d c$ daughter centriole
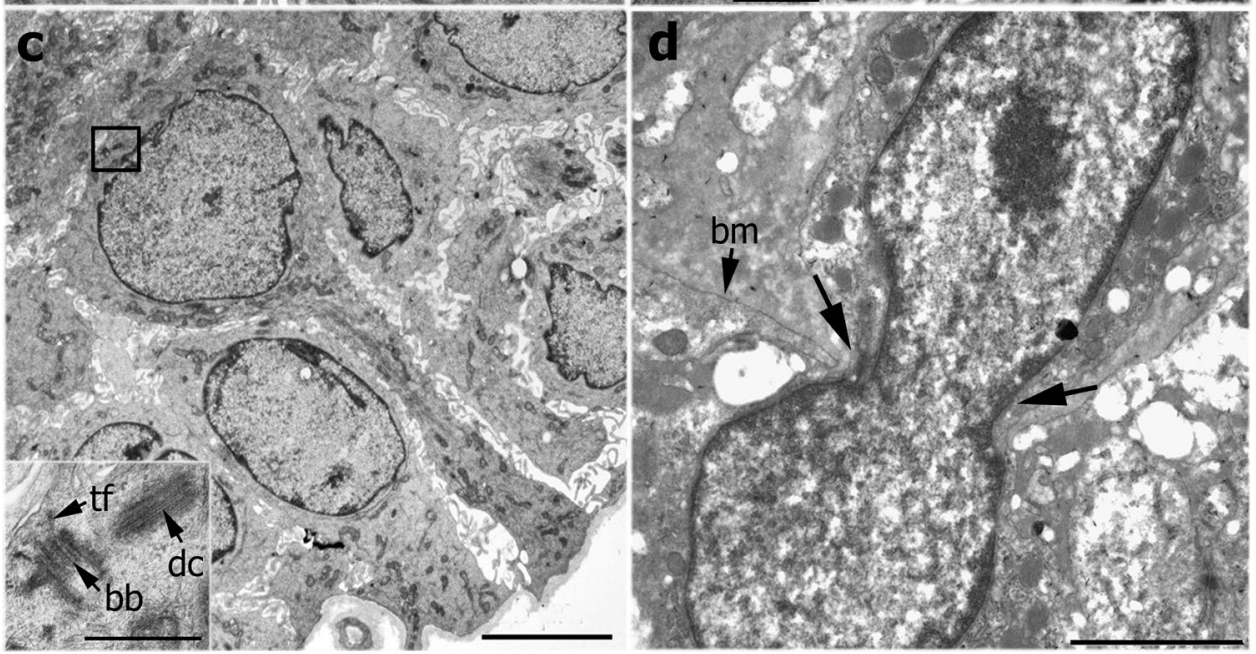
morphology (Fig. 7d). Some epithelial dark cells were found in lamina propria, located near blood vessels (Figs. 6a and $8 \mathrm{a}, \mathrm{c})$. Interestingly, these cells showed primary cilia projecting to the extracellular space, near Golgi apparatus (Fig. 8b,d). These cells kept a typical disposition of organelles in perinuclear area. However, their cytoplasm seemed extremely vacuolated and loss of cytoplasm fragments was found (Fig. 8).

\section{MIBC}

High-grade tumours showed an enormous cellular heterogeneity, although previously described clear and dark cells were still identifiable (Fig. 9a). Abundant mitosis (Fig. 9b) and some apoptotic cells were found (Sup. Fig. 1b). Moreover, tumoral infiltration of muscles, blood vessels and nerves was identified. Clear cells kept their cytological properties, highlighting irregular nuclei and deep invaginations of nuclear envelope (Fig. 9c). These cells established new contacts with clear cells by small punctiform desmosomes and to dark cells through interdigitations generating tumoral cords (Fig. 9c and d). The so-called dark cells were more numerous than in low-grade tumours and, occasionally, they showed a fusiform-mesenchymal morphology (Sup. Fig. 1c). Some cells showed abundant autophagosomes containing rough endoplasmic reticula (Sup. Fig. 1d). Although the exact nature of these structures needs further research, it may point to autophagy impairment in these cells. Moreover, angiogenesis of new blood vessels was identified (Sup. Fig. 1e) and, as pointed above, necrosis and necroptosis were also found in some tumoral cells (Sup. Fig. 1f).

Activated centrioles and primary cilia were also found in MIBC cells (Fig. 9e, f).

Hence, ultrastructure showed that two types of cells were present in bladder cancer: clear and dark cells. The latter showed primary cilia and appeared in NMIBC crossing basement membrane and next to blood vessels. In MIBC, cells show an increased heterogeneity, abundant mitosis, invasiveness and some cellular alterations.
Fig. 8 Tumoral cells crossing basement membrane showed primary cilia. a and $\mathbf{c}$ Some cells showing the ultrastructural features of epithelial tumoral dark cells (i.e., fibrilar nucleoli, decondensed euchromatin, cytoplasmic composition) were found beyond the basement membrane (bm, dashed line) and near blood vessels (bv). These cells present a progressive vacuolization $(\mathrm{v})$ that finally produce cytoplasm release. Interestingly, these cells showed primary cilia. Scale bar $=5 \mu \mathrm{m} \mathbf{b}$ and $\mathbf{d}$ Magnification of squared areas in a and c (respectively) showing a detailed image of primary cilia and their components: basal body $(b b)$, subdistal appendages ( $s a$ ), axoneme ( $a x)$. Near primary cilia, Golgi apparatus is usually present $(\mathrm{g})$. Scale bar $=500 \mathrm{~nm} d c$ daughter centriole, $n$ nuclei, $f f$ transition fibres
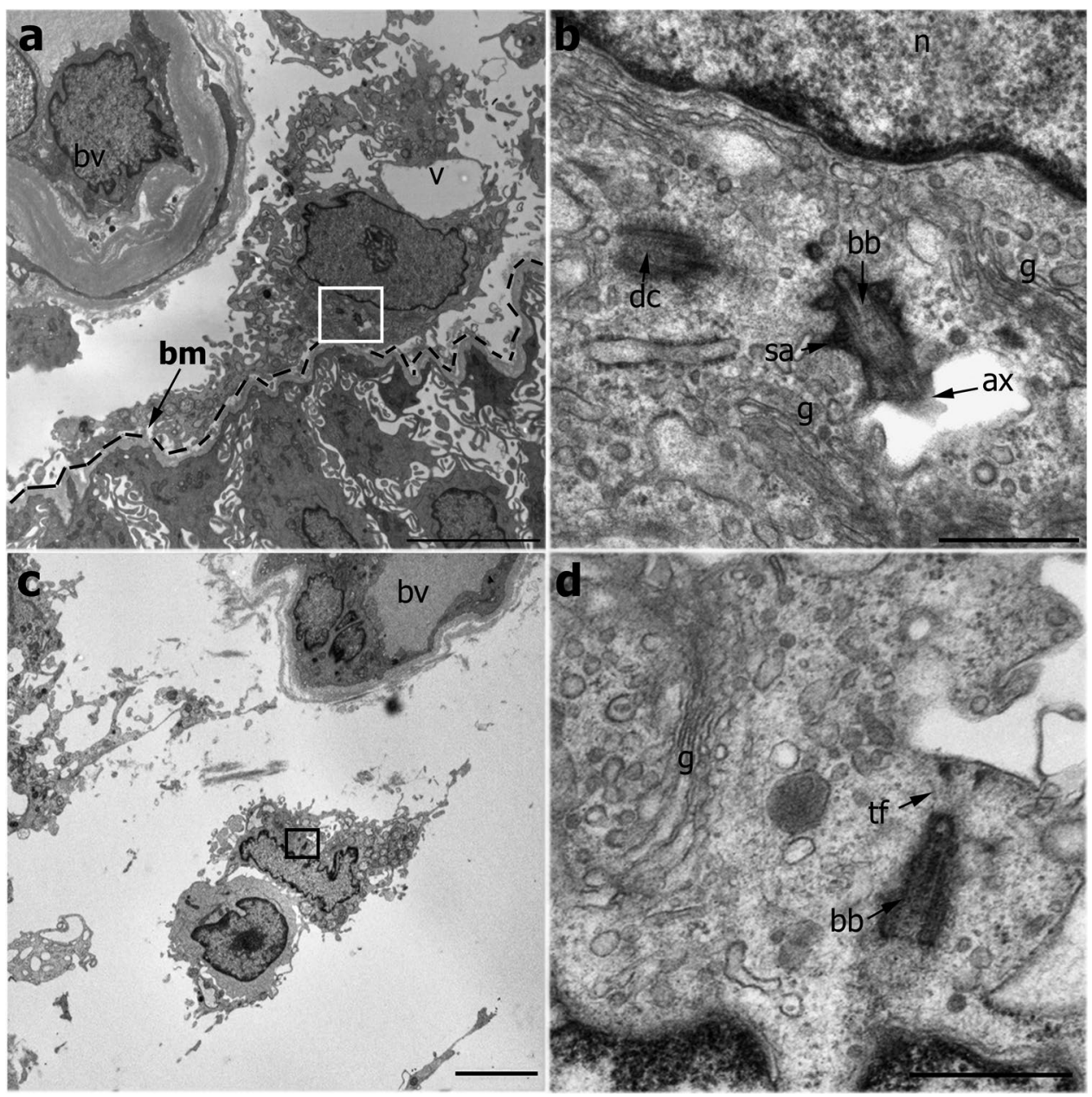
Fig. 9 MIBC ultrastructure. a Heterogenous cells were found in MIBC although dark (d) and clear (c) cells were identifiable. Scale bar $=1 \mu \mathrm{m}$ b Cells in mitosis showing condensed chromosomes separating to the cellular poles. Scale bar $=10 \mu \mathrm{m}$ c Clear cell (c) with a deep nuclear envelope invagination contacting neighbor clear cell (c) by punctual desmosomes (inset). Scale bar $=2 \mu \mathrm{m}$ (inset $500 \mathrm{~nm}$ ) d Contacts between dark cells (d) and clear cells (c) consist of membrane interdigitations (inset). Scale bar $=2 \mu \mathrm{m}$ (inset $500 \mathrm{~nm}$ ) e Activated centrioles with subdistal appendages (sa) and loss of their perpendicular disposition migrating to the cell membrane were found in MIBC cancer cells. Scale bar $=1 \mu \mathrm{m}$ f Dark cells of MIBC samples also present primary cilia (inset). Scale $b a r=1 \mu \mathrm{m}$ (inset $250 \mathrm{~nm}) b b$ basal body, $t f$ transition fibers, $d c$ daughter centriole, $b v$ blood vessel
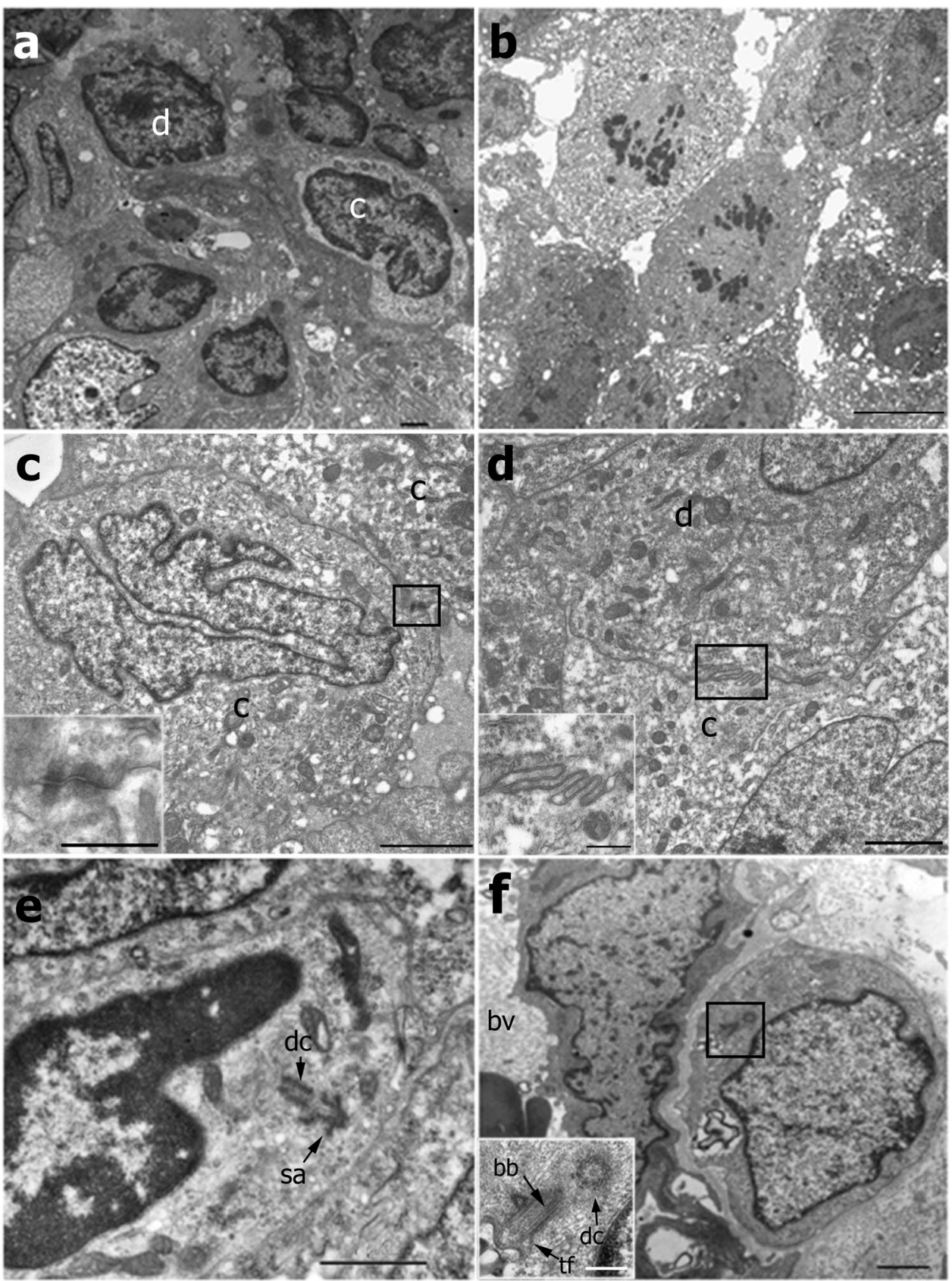

\section{Discussion}

The present study shows new roles and potential implications of primary cilia in bladder cancer, pointing to the potential importance of this structure in tumor progression and invasiveness, likely in relation with epithelial-to-mesenchymal transition (EMT) and the cilia-dependent Hedgehog (Hh) signalling pathway.

EMT is a key element in epithelial tumoral cells, which acquire features related to stemness, invasiveness and metastasis (Wever et al. 2008; Lamouille et al. 2014; Prieto-García et al. 2017). In bladder cancer, the EMT-activator Twist has been related to tumor progression and epithelial markers downregulation (Fondrevelle et al. 2009; Yun and Kim 2013). Accordingly, we found increased Twist expression in MIBC and lower in NMIBC and health controls while the epithelial markers E-cadherin and cytokeratin were downregulated in MIBC. These results confirmed that EMT program is activated in bladder cancer, especially in invasive tumors.

Several studies have demonstrated the importance of $\mathrm{Hh}$ signaling pathway in bladder cancer. However, the exact influence of $\mathrm{Hh}$ activation remains controversial. Most studies point that ligand-dependent Hh signaling activation 
results in increased tumorigenicity, stemness, EMT and migration in bladder cancer (Fei et al. 2012; Islam et al. 2016; Kitagawa et al. 2019). Furthermore, inhibiting Hh has shown to reduce these effects in bladder cancer cells (Islam et al. 2016; Miao et al. 2018; Kitagawa et al. 2019) and to decrease cancer stem cell population (Wang et al. 2017). Conversely, Shin et al. have shown that Hh expression by urothelial basal cells plays a protective role in bladder cancer by stimulating urothelium differentiation through a feedback loop with stromal cells (Shin et al. 2014a). Consequently, they showed $\mathrm{Hh}$ reduction in a mouse model of bladder cancer and human samples (Shin et al. 2014a, b). Our results are in accordance with former works, showing an enhanced expression of Patched, Smo and Gli1 in MIBC. Besides, we did not find Hh pathway components expression in stromal cells or blood vessels as was described in Shin's work (Shin et al. 2014b).

Nevertheless, our experiments interestingly showed that basal cells in some areas of NMIBC presented Hh pathway activation and a reduction of epithelial markers. Our findings suggest that urothelial basal cells are importantly related to EMT and Hh activation in low-grade tumours and probably related to cancer initiation, recurrence and future progression; an idea also shared by other authors (Shin et al. 2014b).

Primary cilia have been straightly related to cancer although their exact role is not completely understood. Hence, it has been related to tumor progression, drugresistance (Jenks et al. 2018), stemness and EMT activation (Guen et al. 2017) especially through Hh signalling (Chahal et al. 2018; Eguether and Hahne 2018). Moreover, primary cilia have been closely related with cell migration both in physiology and pathology (Schneider et al. 2010; Veland et al. 2014), orientating parallel to the movement direction and sensing different signals that regulate cell migration and interacting directly with the extracellular matrix (Veland et al. 2014). Furthermore, this structure also plays an important role in cellular polarity (Tang and Marshall 2012) and its impairment in tumoral epithelial cells.

Primary cilia presence in urothelium has not been defined. Here, we have described for the first time primary cilia in the healthy bladder, NMIBC and MIBC through immunofluorescence and electron microscopy techniques.

Our results show that primary cilia appear on basal cells of healthy controls and NMIBC. In fact, we have interestingly shown that in low-grade tumours, ciliated cells cross basement membrane and invade stroma, which may explain the high recurrent and progression rates found in NMIBC and may point to primary cilia as an important target in these tumours.

The absence of statistical differences in the proportion of ciliated cells in the samples studied would indicate the importance of primary cilia in every tumoral stage in urothelial carcinoma pointing out a specific subpopulation. Thus, we believe this ciliated cellular subpopulation might be related to both, physiologic and cancer stem cells in urothelium as they are in other tissues (Lyu and Zhou 2017). These ciliated basal cells would be related to EMT, tumorogenesis, migration and invasiveness, probably through $\mathrm{Hh}$ pathway activation regardless of the ciliated cell proportion.

One study described a reduction of primary cilia in highgrade bladder cancer regarding non-cancerous tissues (Du et al. 2018). They described that they practically found no ciliated cells in NMIBC and MIBC. Conversely, we found an important number of ciliated cells in tumoral tissues. These opposite results could be a consequence of the heterogeneity found in human tumours, differences in sample processing or the short-length axoneme of urothelial cells which make cilia identification difficult. This short-length axoneme, generally embedded in the ciliary pocket, is in opposition to the long protruding cilia found in other tumours, such as GIST (Castiella et al. 2013). This difference might be in relation to the characteristic location of primary cilia near the cell membrane in epithelial cells (Sorokin 1968; Bernabé-Rubio and Alonso, 2017) and may affect ciliary signalling sensing.

On the other hand, we have described the main ultrastructural elements of EMT in urothelial carcinoma showing loss of cellular unions, formations of filopodia-like structures (which probable mediate cell migration), loss of polarity and enlargement of intercellular space. These processes are in parallel to primary cilia assembly, which remains during advanced stages (probably regulating tumoral progression). To the best of our knowledge, no one has previously described urothelial carcinoma EMT ultrastructural features.

Other ultrastructural findings showed in the present work point out important features of bladder cancer, i.e. ELCS, multivesicular bodies and autophagic vacuoles. ELCS are composed of chromatin fibers sheathed by inner nuclear membrane. Interestingly, they have been related to quiescent brain stem cells and they have been found in different tumours (Olins and Olins 2009; Cebrián-Silla et al. 2017). No one has described this structure in bladder cancer before. Bladder cancer exosomes play an important role in EMT activation and invasiveness (Franzen et al. 2015). Besides, we found abundant autophagosomes containing different organelles, especially rough endoplasmic reticula. Autophagy plays an important role in bladder cancer viability and drug resistance and its inhibition has been shown to increase tumoral cell apoptosis (Lin et al. 2017a, b).

Moreover, it has been described that primary cilia activate autophagy through Hh signaling (Pampliega et al. 2013), suggesting an additional mechanism for primary ciliadependent bladder cancer formation and maintaining.

However, the aim of this work was descriptive and focused on human biopsies to analyse the ultrastructure of these tumours, to confirm previous studies of EMT and $\mathrm{Hh}$ in bladder cancer lines (Islam et al. 2016; Miao et al. 2018; 
Kitagawa et al. 2019) and to give new evidence of primary cilia presence and potential implications. A limitation in this kind of study is the reduced number of samples.

In conclusion, our work sheds light on the importance of primary cilia in bladder cancer showing for the first time the presence of this structure in invasive cells of bladder cancer. Especially interesting is the presence of these ciliated invasive cells in NMBIC, which could explain the high recurrence index of these tumours. Primary cilia may play a fundamental role in basal cells which would initiate tumoral progression and invasiveness even in early tumoral stages probably through EMT and Hh activation. Furthermore, electron microscopy techniques have shown its importance to identify the ultrastructural nature of invading cells, giving evidence of potential targets for bladder cancer treatments.

Acknowledgements Authors would like to acknowledge the use of Servicio General de Apoyo a la Investigación-SAI, Universidad de Zaragoza.

Author contributions PI, EM and CB performed immunohistochemical experiments. TC and GM performed pathological analyses. PI, TC and $\mathrm{CJ}$ performed electron microscopy and its interpretation. PI and CJ wrote the manuscript. TC and CJ designes the study. All the authors read and approved the manuscript.

Funding No funding was specifically received for the experiments shown in this paper.

\section{Compliance with ethical standards}

Conflict of interests The authors declare that they have no conflict of interest.

Ethics approval and consent declarations All protocols and consents developed were approved by the Human Research Ethics Committee "Comité Ético de Investigación Clínica de Aragón".

\section{References}

Amakye D, Jagani Z, Dorsch M (2013) Unraveling the therapeutic potential of the Hedgehog pathway in cancer. Nat Med 19(11):1410-1422. https://doi.org/10.1038/nm.3389

Antoni S et al (2017) 'Bladder cancer incidence and mortality: A global overview and recent trends. Eur Assoc Urol 71(1):96-108. https ://doi.org/10.1016/j.eururo.2016.06.010

Bernabé-Rubio M, Alonso MA (2017) Routes and machinery of primary cilium biogenesis. Cell Mol Life Sci 74(22):4077-4095. https://doi.org/10.1007/s00018-017-2570-5

Bryan RT (2015) Cell adhesion and urothelial bladder cancer: The role of cadherin switching and related phenomena. Philos Trans R Soc B Biol Sci. https://doi.org/10.1098/rstb.2014.0042

Castiella T et al (2013) Primary cilia in gastric gastrointestinal stromal tumours (GISTs): An ultrastructural study. J Cell Mol Med 17(7):844-853. https://doi.org/10.1111/jcmm.12067

Cebrián-Silla A et al (2017) Unique organization of the nuclear envelope in the post-natal quiescent neural stem cells. Stem
Cell Reports 9(1):203-216. https://doi.org/10.1016/j.stemc r.2017.05.024

Chahal KK, Parle M, Abagyan R (2018) Hedgehog pathway and smoothened inhibitors in cancer therapies. Anticancer Drugs. https://doi.org/10.1097/CAD.0000000000000609

Du E et al (2018) Analysis of potential genes associated with primary cilia in bladder cancer. Cancer Manag Res 10:3047-3056. https ://doi.org/10.2147/CMAR.S175419

Eguether T, Hahne M (2018) Mixed signals from the cell's antennae: primary cilia in cancer. EMBO Rep 33:e46589. https://doi. org/10.15252/embr.201846589

Fabbri L, Bost F, Mazure N (2019) Primary cilium in cancer hallmarks. Int J Mol Sci 20:1336. https://doi.org/10.3390/ijms2 0061336

Fei DL et al (2012) Hedgehog signaling regulates bladder cancer growth and tumorigenicity. Cancer Res 15:4449-4459. https:// doi.org/10.1158/0008-5472.CAN-11-4123

Fondrevelle ME et al (2009) The expression of twist has an impact on survival in human bladder cancer and is influenced by the smoking status. Urol Oncol 27(3):268-276. https://doi.org/10.1016/j. urolonc.2007.12.012

Franzen CA et al (2015) Urothelial cells undergo epithelial-to-mesenchymal transition after exposure to muscle invasive bladder cancer exosomes. Oncogenesis 4(8):e163-e210. https://doi.org/10.1038/ oncsis. 2015.21

Global Cancer Observatoty (2018) 'Estimated number of incident cases from 2018 to 2040, liver, both sexes, all ages', p. 800. Available at: http://gco.iarc.fr/tomorrow/graphic-isotype?type=0\&population $=900 \&$ mode $=$ population $\&$ sex $=0 \&$ cancer $=39 \&$ age $\_$group $=$value $\&$ apc $\_$male $=0 \&$ apc_female $=0$

Guen VJ et al (2017) EMT programs promote basal mammary stem cell and tumor-initiating cell stemness by inducing primary ciliogenesis and Hedgehog signaling. Proc Natl Acad Sci. https://doi. org/10.1073/pnas.1711534114

Humphrey PA et al (2016) The 2016 WHO classification of tumours of the urinary system and male genital organs-Part B: prostate and bladder tumours. Eur Assoc Urol 70(1):106-119. https://doi. org/10.1016/j.eururo.2016.02.028

Iruzubieta $\mathrm{P}$ et al (2019) Hedgehog signalling pathway activation in gastrointestinal stromal tumours is mediated by primary cilia. Gastric Cancer. https://doi.org/10.1007/s10120-019-00984-2

Islam S et al (2016) Sonic hedgehog (Shh) signaling promotes tumorigenicity and stemness via activation of epithelial-to-mesenchymal transition (EMT) in bladder cancer. Mol Carcinog 55(5):537-551. https://doi.org/10.1002/mc.22300

Jenks AD et al (2018) Primary cilia mediate diverse kinase inhibitor resistance mechanisms in cancer. Cell Reports 23(10):3042-3055. https://doi.org/10.1016/j.celrep.2018.05.016

Katoh Y, Katoh M (2009) Hedgehog target genes: mechanisms of carcinogenesis induced by aberrant hedgehog signaling activation. Curr Mol Med. https://doi.org/10.2174/156652409789105570

Kitagawa K et al (2019) Possible correlation of sonic hedgehog signaling with epithelial-mesenchymal transition in muscleinvasive bladder cancer progression. J Cancer Res Clin Oncol 145(9):2261-2271. https://doi.org/10.1007/s00432-019-02987-z

Lamouille S, Xu J, Derynck R (2014) Molecular mechanisms of epithelial-mesenchymal transition. Nat Rev Mol Cell Biol 15(3):178196. https://doi.org/10.1038/nrm3758.Molecular

Liedberg $\mathrm{F}$ et al (2015) Local recurrence and progression of nonmuscle-invasive bladder cancer in Sweden: A populationbased follow-up study. Scand J Urol 49(4):290-295. https://doi. org/10.3109/21681805.2014.1000963

Lin JF et al (2017a) Cisplatin induces protective autophagy through activation of BECN1 in human bladder cancer cells. Drug Des Devel Ther 11:1517-1533. https://doi.org/10.2147/DDDT.S1264 64 
Lin YC et al (2017b) Chloroquine and hydroxychloroquine inhibit bladder cancer cell growth by targeting basal autophagy and enhancing apoptosis. Kaohsiung J Med Sci 33(5):215-223. https://doi. org/10.1016/j.kjms.2017.01.004

Lyu R, Zhou J (2017) The multifaceted roles of primary cilia in the regulation of stem cell properties and functions. J Cell Physiol 232(5):935-938. https://doi.org/10.1002/jcp.25683

Malicki JJ, Johnson CA (2017) The cilium: cellular antenna and central processing unit. Trends Cell Biol 27(2):126-140. https://doi. org/10.1016/j.tcb.2016.08.002

Miao X et al (2018) Down-regulation of microRNA-224 -inhibits growth and epithelial-to-mesenchymal transition phenotype -via modulating SUFU expression in bladder cancer cells. Int J Biol Macromol 106:234-240. https://doi.org/10.1016/j.ijbio mac.2017.07.184

Nedjadi T et al (2019) Sonic hedgehog expression is associated with lymph node invasion in urothelial bladder cancer. Pathol Oncol Res 25(3):1067-1073. https://doi.org/10.1007/s12253-018-0477-6

Olins DE, Olins AL (2009) Nuclear envelope-limited chromatin sheets (ELCS) and heterochromatin higher order structure. Chromosoma 118(5):537-548. https://doi.org/10.1007/s00412-009-0219-3

Pampliega $\mathrm{O}$ et al (2013) Functional interaction between autophagy and ciliogenesis. Nature 502(7470):194-200. https://doi.org/10.1038/ nature 12639

Prieto-García E et al (2017) Epithelial-to-mesenchymal transition in tumor progression. Med Oncol. https://doi.org/10.1007/s1203 2-017-0980-8

Ramsbottom SA et al (2016) Regulation of hedgehog signalling inside and outside the cell. J Dev Biol. https://doi.org/10.3390/jdb40 30023. Regulation

Rohatgi R, Milenkovic L, Scott MP (2007) Patched1 regulates hedgehog signaling at the primary cilium. Science 317(5836):372-376. https://doi.org/10.1126/science.1139740

Schindelin J et al (2012) Fiji: an open-source platform for biological-image analysis. Nat Methods 9(7):676-682. https://doi. org/10.1038/nmeth.2019

Schneider L et al (2010) Directional cell migration and chemotaxis in wound healing response to PDGF-AA are coordinated by the primary cilium in fibroblasts. Cell Physiol Biochem 25(2-3):279292. https://doi.org/10.1159/000276562
Shin K, Lim A, Zhao C et al (2014b) Hedgehog signaling restrains bladder cancer progression by eliciting stromal production of urothelial differentiation factors. Cancer Cell 26(4):521-533. https ://doi.org/10.1016/j.ccell.2014.09.001

Shin K, Lim A, Odegaard JI et al (2014a) Cellular origin of bladder neoplasia and tissue dynamics of its progression to invasive carcinoma. Nat Cell Biol. https://doi.org/10.1038/ncb2956

Sorokin SP (1968) Centriole formation and ciliogenesis. Aspen Emphysema Conf 11:213-216

Tang N, Marshall WF (2012) Centrosome positioning in vertebrate development. J Cell Sci 125(21):4951-4961. https://doi. org/10.1242/jcs.038083

Veland IR, Lindbæk L, Christensen ST (2014) Linking the primary cilium to cell migration in tissue repair and brain development. Bioscience 64(12):1115-1125. https://doi.org/10.1093/biosci/ biu179

Wang D et al (2017) Curcumin inhibits bladder cancer stem cells by suppressing Sonic Hedgehog pathway. Biochem Biophys Res Commun 493(1):521-527. https://doi.org/10.1016/j. bbrc.2017.08.158

De WO et al (2008) 'Molecular and pathological signatures of epithelial-mesenchymal transitions at the cancer invasion front. Histochem Cell Biol. https://doi.org/10.1007/s00418-008-0464-1

Wheway G, Nazlamova L, Hancock JT (2018) Signaling through the primary cilium. Front Cell Develop Biol 6:1-13. https://doi. org/10.3389/fcell.2018.00008

Yun SJ, Kim W (2013) 'Role of the epithelial-mesenchymal transition in bladder cancer: from prognosis to therapeutic target. Korean J Urol 54:645-650

Zeisberg M, Neilson EG (2009) Review series personal perspective Biomarkers for epithelial-mesenchymal transitions. J Clin Invest 119(6):1429-1437. https://doi.org/10.1172/JCI36183.protected

Publisher's Note Springer Nature remains neutral with regard to jurisdictional claims in published maps and institutional affiliations. 